\title{
Lattice dynamics and vibrational spectra of the orthorhombic, tetragonal, and cubic phases of methylammonium lead iodide
}

\author{
Federico Brivio, ${ }^{1}$ Jarvist M. Frost, ${ }^{1}$ Jonathan M. Skelton, ${ }^{1}$ Adam J. Jackson, ${ }^{1}$ Oliver J. Weber, ${ }^{1}$ Mark T. Weller, ${ }^{1}$ \\ Alejandro R. Goñi, ${ }^{2}$ Aurélien M. A. Leguy, ${ }^{3}$ Piers R. F. Barnes, ${ }^{3}$ and Aron Walsh ${ }^{1,4, *}$ \\ ${ }^{1}$ Centre for Sustainable Chemical Technologies and Department of Chemistry, University of Bath, Claverton Down, \\ Bath BA2 7AY, United Kingdom \\ ${ }^{2}$ ICREA, Passeig Lluís Companys 23, E-08010 Barcelona, Spain \\ and Institut de Ciència de Materials de Barcelona (ICMAB-CSIC), Campus UAB, E-08193 Bellaterra, Spain \\ ${ }^{3}$ Department of Physics, Imperial College London, London SW7 2AZ, United Kingdom \\ ${ }^{4}$ Global $E^{3}$ Institute and Department of Materials Science and Engineering, Yonsei University, Seoul 120-749, Korea
}

(Received 30 May 2015; revised manuscript received 21 September 2015; published 16 October 2015)

\begin{abstract}
The hybrid halide perovskite $\mathrm{CH}_{3} \mathrm{NH}_{3} \mathrm{PbI}_{3}$ exhibits a complex structural behavior, with successive transitions between orthorhombic, tetragonal, and cubic polymorphs around 165 and $327 \mathrm{~K}$. Herein we report first-principles lattice dynamics (phonon spectrum) for each phase of $\mathrm{CH}_{3} \mathrm{NH}_{3} \mathrm{PbI}_{3}$. The equilibrium structures compare well to solutions of temperature-dependent powder neutron diffraction. By following the normal modes, we calculate infrared and Raman intensities of the vibrations, and compare them to the measurement of a single crystal where the Raman laser is controlled to avoid degradation of the sample. Despite a clear separation in energy between low-frequency modes associated with the inorganic $\left(\mathrm{PbI}_{3}{ }^{-}\right)_{n}$ network and high-frequency modes of the organic $\mathrm{CH}_{3} \mathrm{NH}_{3}{ }^{+}$cation, significant coupling between them is found, which emphasizes the interplay between molecular orientation and the corner-sharing octahedral networks in the structural transformations. Soft modes are found at the boundary of the Brillouin zone of the cubic phase, consistent with displacive instabilities and anharmonicity involving tilting of the $\mathrm{PbI}_{6}$ octahedra around room temperature.
\end{abstract}

DOI: 10.1103/PhysRevB.92.144308

\section{INTRODUCTION}

Materials that adopt the perovskite crystal structure are known for their complex structural landscapes, with a large number of accessible polymorphs depending on the temperature, pressure, and/or applied electric field. For ternary $A B X_{3}$ perovskites, the $A$ site cation is at the center of a cube formed of corner-sharing $B X_{6}$ octahedra. Displacement of the $A$ cation is usually associated with a ferroelectric (Brillouin-zone center) instability, while tilting of the $B X_{6}$ octahedral network is usually linked to antiferroelectric (Brillouin-zone boundary) transitions [1,2].

Hybrid organic-inorganic perovskites are formed when one of the elemental perovskite building blocks is replaced by a molecular anion or cation [3,4]. There exists a large family of such compounds, including the widely studied formate perovskites, which contains both molecular anions and cations [5-7]. Hybrid halide perovskites are of current intense research effort due to their high-efficiency photovoltaic action [8-16].

Methylammonium lead iodide $\left(\mathrm{MAPbI}_{3}\right.$, where MA represents the $\mathrm{CH}_{3} \mathrm{NH}_{3}{ }^{+}$cation) was first reported by Weber in 1978 [17]. It is the most relevant hybrid halide perovskite for photovoltaic application. The transition from orthorhombic to tetragonal to cubic perovskite structures as a function of temperature has been studied by techniques including

\footnotetext{
*a.walsh@bath.ac.uk

Published by the American Physical Society under the terms of the Creative Commons Attribution 3.0 License. Further distribution of this work must maintain attribution to the author(s) and the published article's title, journal citation, and DOI.
}

PACS number(s): 78.30.-j, 63.20.D-, 63.20.Ry

calorimetry and infrared spectroscopy [18], single-crystal xray diffraction [19], and dielectric spectroscopy [20]. Recently analysis of powder neutron-diffraction (PND) measurements has provided more quantitative insight into the temperaturedependent behavior of the MA cation within the anionic $\left(\mathrm{PbI}_{3}{ }^{-}\right)_{n}$ network [21]. There is now direct evidence for the degree of order of the MA cation in the different phases, and the average lattice parameters (and thus extent of octahedral tilting) as a function of temperature through the first- and second-order phase transitions. Quasielastic neutron scattering has provided further insight into the rotational dynamics of the MA cation with a room-temperature residence time of $\sim 14$ ps [22], while time-resolved vibrational spectroscopy has identified fast librations (300 fs) and slow rotations (3 ps) of the molecule [23].

In this study, we calculate the phonon dispersion in each phase of $\mathrm{MAPbI}_{3}$ within the harmonic approximation, computing the force constants with density functional theory (DFT). We use the PBEsol functional, which is a generalized-gradient approximation (GGA) to the exchange-correlation functional, numerically evaluated with Perdew's method, adjusted to give more accurate lattice constants and forces for solids [24]. The lattice dynamic calculations allow the atomic origin of each phonon mode to be identified. Changes in lattice polarization and polarizability for each eigenvector provide the infrared and Raman activity of each mode. Spectral features related to the inorganic and organic components (from 0 to $3000 \mathrm{~cm}^{-1}$ ) are well reproduced in comparison to the Raman spectra of a single crystal of $\mathrm{MAPbI}_{3}$. Overlap is found between the vibrations of the $\mathrm{CH}_{3} \mathrm{NH}_{3}{ }^{+}$and $\mathrm{PbI}_{3}{ }^{-}$components up to $130 \mathrm{~cm}^{-1}$, with the modes from 300 to $3000 \mathrm{~cm}^{-1}$ being associated with pure molecular vibrations. The phonon dispersion has implications for developing quantitative models for the generation, 
transport, and recombination of photogenerated electrons and holes in hybrid perovskite solar cells.

\section{Structure models}

The normal modes of a system are defined for an equilibrium configuration. Calculating the vibrations for a nonequilibrium structure will result in imaginary frequencies upon diagonalizing the dynamical matrix. Therefore, we have generated well-optimized structures of $\mathrm{MAPbI}_{3}$. One challenge in calculating the phonons of hybrid perovskites is the soft nature and complicated potential-energy landscape of some of the restoring potentials, particularly those involving the organic cation.

The models for the crystal structures used in this study are discussed in detail below and a comparison with the measured diffraction patterns is provided as Supplemental Material [25].

\section{Orthorhombic phase}

The orthorhombic perovskite structure is the lowtemperature ground state of $\mathrm{MAPbI}_{3}$ and maintains its stability up to around $165 \mathrm{~K}[21,26,27]$. A comparison of the enthalpy from DFT calculations confirms this ordering in stability. The difference in enthalpy is small, i.e., just $2 \mathrm{meV}$ per $\mathrm{MAPbI}_{3}$ unit, compared to the lowest energy tetragonal phase, yet $90 \mathrm{meV}$ compared to the high-temperature cubic phase.

Initial diffraction pattern solutions are assigned to the $P n a 2_{1}$ space group [18,26]. Recent analysis of higher-quality powder neutron-diffraction data reassigns it to Pnma (a $D_{2 h}$ point group) [21]. The structure is a $\sqrt{2} a \times \sqrt{2} a \times 2 a$ supercell expansion of the simple cubic perovskite lattice, i.e., following the lattice transformation matrix

$$
\left(\begin{array}{ccc}
1 & -1 & 0 \\
1 & 1 & 0 \\
0 & 0 & 2
\end{array}\right) .
$$

In the $P n a 2_{1}$ phase, the $\mathrm{PbI}_{6}$ octahedra are distorted and tilt as $a^{+} b^{-} b^{-}$in Glazer notation [1] with respect to the orientation of the conventional cubic cell. In this low-temperature phase, the four molecular cations in the unit cell are static on the diagonals of the $a b$ planes pointing towards the undistorted facets of the cuboctahedral cavity. Correspondingly, molecules belonging to different planes are antialigned with a head-tail motif. Such an antiferroelectric alignment is expected from consideration of the molecular dipole-dipole interaction [28].

In the low-temperature orthorhombic phase, the $\mathrm{CH}_{3} \mathrm{NH}_{3}{ }^{+}$ sublattice is fully ordered (a low-entropy state). The ordering may be sensitive to the material preparation and/or cooling rate into this phase, i.e., the degree of quasithermal equilibrium. It is possible that different ordering might be frozen into the low-temperature phase by epitaxy or application of external force or electric fields.

\section{Tetragonal phase}

At $165 \mathrm{~K}, \mathrm{MAPbI}_{3}$ goes through a first-order phase transition from the orthorhombic to the tetragonal space group $I 4 / \mathrm{mcm}$ ( $D_{4 h}$ point group), which continuously undergoes a second-order phase transition to the cubic phase around $327 \mathrm{~K}[21,26,27]$. As with the orthorhombic phase, this can be considered a $\sqrt{2} a \times \sqrt{2} a \times 2 a$ expansion of the cubic perovskite unit cell.

The molecular cations are no longer in a fixed position as in the orthorhombic phase. The molecules are disordered between two nonequivalent positions in each cage [27,30]. The tetragonal distortion parameter in the cubic basis is greater than unity $\left(\frac{c}{2 a} \sim 1.01\right.$ at $\left.300 \mathrm{~K}\right)$, corresponding to an elongation of the $\mathrm{PbI}_{6}$ octahedra along the $c$ axis. The associated octahedral tilting pattern is $a^{0} a^{0} c^{-}$in Glazer notation.

Atomistic simulations within periodic boundary conditions require an ordered configuration. The solved crystal structure shows that there are several possible configurations for the organic cations within the tetragonal unit cell. These configurations have similar enthalpies within DFT [31], which is consistent with the observed disorder. We choose to use the most energetically stable structure, which is also consistent with a previous DFT investigation [32].

In the model of the tetragonal structure, the MA cations are aligned as in the orthorhombic phase, towards the face of the perovskite cage, i.e., $\langle 100\rangle$ in the cubic basis. The MA in different (001) planes are approximately orthogonal to one another. The orientational dynamics of the methylammonium ions, which exists above $165 \mathrm{~K}$, is not taken into account in this equilibrium configuration study.

\section{Cubic phase}

With increasing temperature, the tetragonal lattice parameters become more isotropic (i.e., $\frac{c}{2 a}$ moves closer to 1 ) and the molecular disorder increases, to the point where a transition to a cubic phase occurs around $327 \mathrm{~K}$. The transition can be seen clearly from changes in the heat capacity [18] as well as in temperature-dependent neutron diffraction [21].

The cubic space group $P m \overline{3} m$ ( $O_{h}$ symmetry) has been assigned to this high-temperature phase. Although the methylammonium ions possesses $C_{3 v}$ symmetry, the orientational disorder gives rise to the effective higher symmetry on average. The local structure will necessarily have a lower symmetry. Indeed, for the bromide and chloride analogues of $\mathrm{MAPbI}_{3}$, pair-distribution function analysis of x-ray scattering data indicates a local structure with significant distortion of the lead halide framework at room temperature [33].

We previously considered alignment of the molecules along the principal $\langle 100\rangle$ (face), $\langle 110\rangle$ (edge), and $\langle 111\rangle$ (diagonal) directions of the cubic unit cell, and showed that they are of similar DFT enthalpy, with a small barrier for rotation [34]. Further $a b$ initio molecular dynamics showed an average preference for the $\langle 100\rangle$ facial configuration at $300 \mathrm{~K}$ [28]. Therefore, we chose the $\langle 100\rangle$ configuration as our reference structure for the lattice vibrations.

Representations of the crystal structure of each phase are shown in Fig. 1, the equilibrium structure parameters are listed in Table I, and the structures themselves are available in an online repository [29].

\section{METHODS}

\section{A. Computations}

The total energy and atomic forces were computed from first principles within density functional theory as implemented 


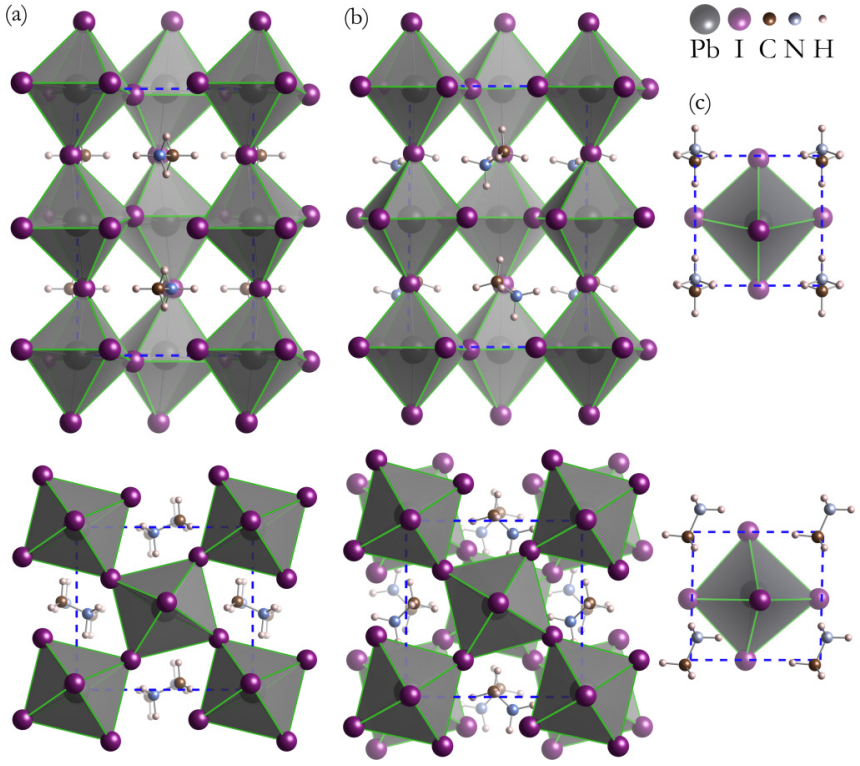

FIG. 1. (Color online) The crystal structures of the (a) orthorhombic, (b) tetragonal, and (c) cubic phases of $\mathrm{CH}_{3} \mathrm{NH}_{3} \mathrm{PbI}_{3}$. The upper and lower panels are oriented through $\langle 100\rangle$ and $\langle 001\rangle$, respectively. Lattice parameters and coordinates obtained from powder neutron diffraction were optimized using density functional theory (PBEsol). The $\mathrm{PbI}_{6}$ octahedra are shaded gray. All structures are available in an online repository [29].

in the code VASP [36,37]. Noise in the lattice vibrations was minimized by rigorous convergence of total Kohn-Sham energy with respect to the basis set (kinetic-energy cutoff for plane waves) and sampling of reciprocal space (density of the $k$-point mesh). The final computational setup is summarized in Table I.

We performed complete optimization of the cell volume, shape, and atomic positions, with the PBEsol [24] semilocal exchange-correlation functional. The scalar-relativistic projector-augmented wave method [38] was employed, with a pseudopotential treating the $\mathrm{Pb} 5 d$ orbitals as valence. Spin-orbit coupling was not considered as it mainly affects the $\mathrm{Pb} 6 p$ conduction band, which does not influence the interatomic interactions at equilibrium. All atomic forces were reduced to below a threshold of $1 \mathrm{meV} / \AA$. Due to the presence of the organic cations, which break the ideal lattice symmetry, deviations in the expected parameters can occur, e.g., in the high-temperature pseudocubic phase, the three equilibrium lattice parameters are not equal. The equilibrium structure parameters (at $0 \mathrm{~K}$ and excluding zero-point contributions) are reported in Table $\mathrm{I}$.

The normal modes are calculated within the harmonic approximation, using the PHONOPY [39-41] package to construct and evaluate the dynamical matrix composed of DFT force constants. Both the finite displacement method (FDM or supercell approach) [42] and density functional perturbation theory (DFPT) [43] approaches to construct the force constants were tested. The results of both approaches produced similar vibrational spectra, with a variance in the mode energies of $6 \mathrm{~cm}^{-1}$.

Within a primitive cell of $N$ atoms, there are $6 N$ possible displacements $( \pm x, \pm y, \pm z)$, which can be reduced by the crystal symmetry. For the orthorhombic phase, the 288 possible displacements are reduced to 41 , while the tetragonal and cubic phases required 288 and 72 displacements, respectively. The phonon dispersion (for $\mathbf{q}$ points away from the Brillouin-zone center, the $\Gamma$ point) in the cubic phase was probed in a $2 \times 2 \times 2$ supercell. Due to computational expense, we did not calculate this for the other (larger unit cell) phases, where the phonons are considered at the $\Gamma$ point only.

Once the normal eigenmodes and eigenvalues are calculated, it is possible to model their associated Raman and infrared (IR) activity by mode following. The two spectroscopic techniques probe different physical responses of the material: the change in polarization for IR and the change in polarizability for Raman. The IR spectra are simulated with the analytic formula of Gianozzi and Baroni (using the Born effective charge tensor) [43]. Prediction of the Raman spectra required computing the change in macroscopic dielectric tensor with respect to each normal mode of the system, a significant DFT calculation in terms of computational expense [44].

\section{B. Experiment}

Methylammonium lead iodide single crystals were grown according to the method of Poglitsch and Weber [26]. $12.5 \mathrm{~g}$ of lead acetate trihydrate $\left[\mathrm{Pb}\left(\mathrm{CH}_{3} \mathrm{CO}_{2}\right)_{2} \cdot 3 \mathrm{H}_{2} \mathrm{O}\right.$, Sigma $]$ was

TABLE I. Equilibrium cell parameters from DFT/PBEsol energy minimization, including the converged plane-wave cutoff, $k$-point mesh, and force threshold. $\mathrm{Z}$ represents the number of formula units of $\mathrm{CH}_{3} \mathrm{NH}_{3} \mathrm{PbI}_{3}$ per cell. The calculated difference in enthalpy $(\Delta \mathrm{H})$ of each phase is given with respect to the ground-state orthorhombic configuration and per $\mathrm{CH}_{3} \mathrm{NH}_{3} \mathrm{PbI}_{3}$ unit. Shown for comparison are the cell parameters and average $\mathrm{Pb}$-I interatomic separations $(d)$ from powder neutron diffraction (PND) [21].

\begin{tabular}{|c|c|c|c|c|c|c|c|c|c|}
\hline Phase & $a(\AA)$ & $b(\AA)$ & $c(\AA)$ & $d(\mathrm{~Pb}-\mathrm{I})(\AA)$ & $Z$ & Cutoff $(\mathrm{eV})$ & $k$ points & Forces $(\mathrm{meV} / \AA)$ & $\Delta \mathrm{H}(\mathrm{meV})$ \\
\hline \multicolumn{10}{|l|}{ Orthorhombic } \\
\hline DFT/PBEsol & 9.04 & 12.66 & 8.35 & 3.18 & 4 & 700 & $5 \times 4 \times 5$ & 1 & 0 \\
\hline PND $(100 \mathrm{~K})$ & 8.87 & 12.63 & 8.58 & 3.19 & & & & & \\
\hline \multicolumn{10}{|l|}{ Tetragonal } \\
\hline DFT/PBEsol & 8.70 & 8.72 & 12.83 & 3.19 & 4 & 800 & $5 \times 5 \times 3$ & 1 & 2 \\
\hline PND (180 K) & 8.81 & 8.81 & 12.71 & 3.17 & & & & & \\
\hline \multicolumn{10}{|l|}{ Cubic } \\
\hline DFT/PBEsol & 6.29 & 6.23 & 6.37 & 3.17 & 1 & 700 & $6 \times 6 \times 6$ & 1 & 90 \\
\hline PND (352 K) & 6.32 & 6.32 & 6.32 & 3.16 & & & & & \\
\hline
\end{tabular}


dissolved in $10 \mathrm{~mL}$ hydroiodic acid ( $\mathrm{HI}_{a q}, 57 \mathrm{wt} \%$, Sigma) in a $50 \mathrm{~mL}$ round bottom flask and heated to $100{ }^{\circ} \mathrm{C}$ in an oil bath. Separately, $0.597 \mathrm{~g}$ of $\mathrm{CH}_{3} \mathrm{NH}_{2}$ (aq, $40 \mathrm{wt} \%$, Sigma) was added dropwise to a further $2 \mathrm{~mL}$ of $\mathrm{HI}_{a q}$ kept at $0{ }^{\circ} \mathrm{C}$ in an ice bath under stirring. The methylammonium iodide solution was then added to the lead acetate solution and the mixture was cooled over five days to a temperature of $46^{\circ} \mathrm{C}$, resulting in the formation of black crystals with largest face length around $8 \mathrm{~mm}$. The content of the flasks was subsequently filtered and dried for 12 hours at $100^{\circ} \mathrm{C}$.

Raman spectra were collected in backscattering geometry with a high-resolution LabRam HR800 spectrometer using a grating with 600 lines per millimeter and equipped with a liquid-nitrogen-cooled charge coupled device (CCD) detector. The $785 \mathrm{~nm}$ line of a diode-pumped solid-state laser was used as excitation beam and focused onto the sample using a longdistance $20 \times$ microscope objective. Raman measurements were carried out at $100 \mathrm{~K}$ using a gas-flow-type cryostat with optical access that fits under the microscope of the Raman setup. The high spectral resolution and stray-light rejection of the LabRam spectrometer, particularly in combination with the $785 \mathrm{~nm}$ line, allowed us to measure the Raman spectrum of MAPI at very low Raman shifts down to 20 to $30 \mathrm{~cm}^{-1}$. In this way, we were able to spectrally resolve several low-frequency modes associated with vibrations of the inorganic cage of the hybrid perovskite.

Heating by laser light directly absorbed by $\mathrm{CH}_{3} \mathrm{NH}_{3} \mathrm{PbI}_{3}$ has been shown to lead to rapid degradation of the material, resulting in $\mathrm{PbI}_{2}$ Raman signatures [45]. Since $785 \mathrm{~nm}$ light is only weakly absorbed, the heating effect of the laser was low enough to ensure the crystal structure was preserved. The power density incident on the sample was kept at $80 \mathrm{~W} / \mathrm{cm}^{2}$. At such power level, it was checked that no appreciable spectral changes in peak width and/or position occurred, while still providing a good signal-to-noise ratio. Further, samples were kept under vacuum inside the cryostat during the measurements.

\section{RESULTS}

\section{A. Harmonic phonons}

The full phonon density of states (DOS) is shown for the three phases of $\mathrm{CH}_{3} \mathrm{NH}_{3} \mathrm{PbI}_{3}$ in Fig. 2. Also plotted is the partial DOS, where assignment to $\mathrm{CH}_{3} \mathrm{NH}_{3}{ }^{+}$or $\mathrm{PbI}_{3}{ }^{-}$ is performed based on the atomic contribution to each eigenvector. An animation of all 36 eigenmodes of the cubic phase is provided as Supplemental Material [25].

Qualitatively, each $\mathrm{MAPbI}_{3}$ phase shows similar vibrational properties with three energetic regions of phonons: (i) a lowfrequency band from $0-150 \mathrm{~cm}^{-1}$, (ii) a midfrequency band from 280-1600 $\mathrm{cm}^{-1}$, and (iii) a high-frequency band from $2900-3300 \mathrm{~cm}^{-1}$. These ranges are consistent with previous computational reports [46,47].

Due to the large difference in atomic mass of the organic and inorganic components, and to the difference in bonding between the inorganic cage and the covalently bonded molecule, we anticipated that the low-frequency modes would be comprised entirely of motion by the $\mathrm{PbI}_{6}$ octahedra, while the high-frequency modes would involve the $\mathrm{CH}_{3} \mathrm{NH}_{3}{ }^{+}$cation.
This is qualitatively the case, but there is also significant coupling between the two. Taking the example of the cubic phase with 36 modes, the highest-energy 18 modes [forming bands (ii) and (iii)] correspond to molecular vibrations, i.e., the $3 N-6$ modes of the methylammonium ion. For an isolated nonlinear molecule, the 6 translational and rotational degrees of freedom do not contribute to the pure vibrational spectrum, but this is not the case for a molecule inside a cuboctahedral cavity.

The 6 additional molecular modes are strongly coupled to the 9 modes (i.e., $3 N-3$ for $\mathrm{PbI}_{3}{ }^{-}$) associated with stretching of the $\mathrm{Pb}$-I bonds and breathing of the $\mathrm{PbI}_{6}$ octahedra, which results in the spectral overlap observed in the partial DOS of band (i). Particularly striking is the low-frequency pivoting motion associated with the libration of the molecular dipole, coupled with a breathing of the octahedral framework (e.g., modes 10 and 15 in the SI). The final three zero-frequency modes correspond to acoustic translations of the lattice.

\section{B. Vibrational spectra}

For $\mathrm{MAPbI}_{3}$, it is not possible for the relaxed (nonidealized) crystal structures to assign the spectral activity directly with group theoretic irreducible representation analysis of the phonon modes. The molecule breaks the average crystal symmetry. This symmetry lowering allows for simultaneous Raman and IR activity, even in the pseudocubic phase. The predicted spectra (the $\Gamma$-point phonon modes weighted by the computed spectral intensity, convolved with a Lorentzian for experimental comparison) are reported in Fig. 2 for each phase.

Raman and IR activity is observed across each of the three phonon bands previously discussed. A notable exception is the lowest-energy purely molecular vibration near $300 \mathrm{~cm}^{-1}$, which is neither Raman nor IR active.

To understand the effect of embedding the methylammonium in $\mathrm{MAPbI}_{3}$, we calculate the normal-mode vibrations of an isolated methylammonium ion in vacuum with a similar density functional theory method to our periodic calculations Perdew-Burke-Ernzerhof (PBE) with an atom-centered augmented cc-pVQZ basis set. Thereby, we calculate the 18 molecular modes, directly accessing their symmetries and nature. The $C_{3 v}$ symmetry of the molecule separates the vibrational bands into one $A$ symmetric mode and blueshifted twofold degenerate asymmetric $E$ modes. The six bands we find are in ascending energy: twist around the $\mathrm{C}-\mathrm{N}$ axis (282, $\left.886 \mathrm{~cm}^{-1}\right)$, vibration along the $\mathrm{C}-\mathrm{N}$ axis $\left(923,1239 \mathrm{~cm}^{-1}\right)$, bending of the $\mathrm{C}-\mathrm{H}$ bonds $\left(1418,1451 \mathrm{~cm}^{-1}\right)$, bending of the $\mathrm{N}-\mathrm{H}$ bonds $\left(1478,1622 \mathrm{~cm}^{-1}\right)$, stretching of the $\mathrm{C}-\mathrm{H}$ bonds $\left(3018,3119 \mathrm{~cm}^{-1}\right)$, and stretching of the N-H bonds $(3321$, $\left.3395 \mathrm{~cm}^{-1}\right)$.

As the cation charge density is centered towards the $\mathrm{N}$, the motion of the protons associated with $\mathrm{N}$ have the strongest affect on the dipole moment and therefore strongest IR activity. Due to the stronger bonds, their frequencies are consistently blueshifted relative to the $\mathrm{C}$ end. Owing to the molecular dipole moment (2.2 D [28], which is rotation and position invariant, and corresponds to a polarization contribution of $\sim 3 \mu \mathrm{C} / \mathrm{cm}^{2}$ ), the two high-frequency asymmetric stretching modes of $\mathrm{NH}_{3}{ }^{+}$ [band (iii)] results in the strongest absolute IR intensity. The hydrogen stretching modes [band (iii)] are responsible for 
- DOS $-\mathrm{CH}_{3} \mathrm{NH}_{3}{ }^{+}$(Cation) $\mathrm{Pb} / \mathrm{I}($ Cage $)-\mathrm{IR}-$ Raman $-\quad$ Exp. IR (RT) - Exp. Raman (100 K)
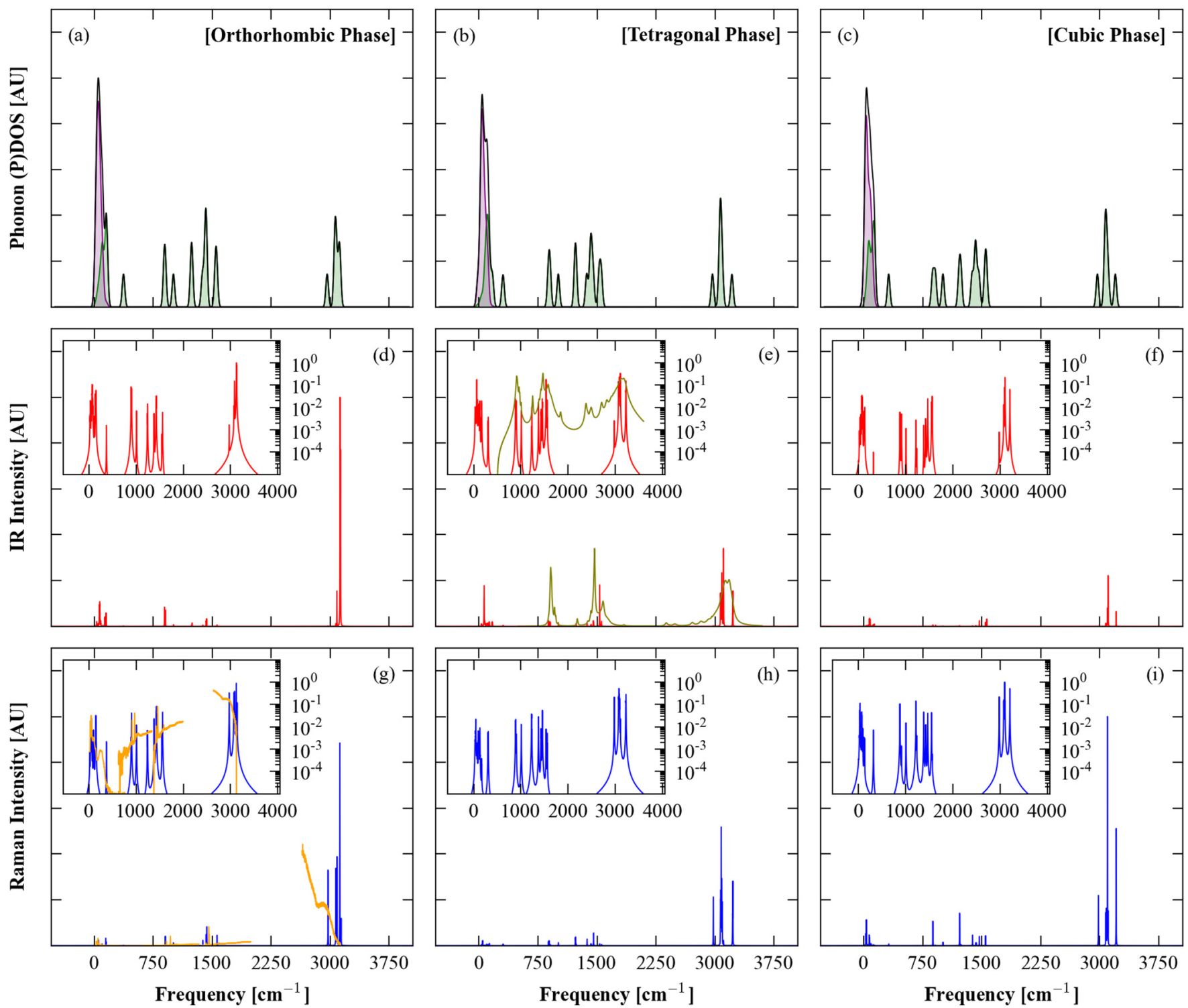

FIG. 2. (Color online) (a)-(c) Projected phonon density of states (PDOS) for the three phases of $\mathrm{CH}_{3} \mathrm{NH}_{3} \mathrm{PbI}_{3}$ as calculated from DFT/PBEsol, generated by convolution with a $16 \mathrm{~cm}^{-1}$ Lorentzian. Note that no imaginary (negative) modes are found in the orthorhombic or tetragonal phases. (d)-(f) Simulated infrared (IR) spectra. (e) The measured IR spectrum at 300 K from Ref. [35] is coplotted in green (light gray). (g)-(i) Simulated Raman spectrum. (g) The measured Raman spectrum of a single crystal in the orthorhombic phase at $100 \mathrm{~K}$ is coplotted in orange (light gray). Simulated spectra were broadened by convolution with a $2 \mathrm{~cm}^{-1}$ Lorentzian. Insets in the simulated spectra are the same data on a logarithmic scale to show the structure in the low-intensity modes.

significant Raman activity. The only mode involving $\mathrm{C}$ or $\mathrm{N}$ motion is the weakly IR and Raman active vibration at $923 \mathrm{~cm}^{-1}$ (vacuum), $1007 \mathrm{~cm}^{-1}$ (cubic perovskite).

The rotation of the $\mathrm{CH}_{3}$ against the $\mathrm{NH}_{3}$ unit, while being strongly populated in molecular dynamic simulations, and which forms the main source of quasi-inelastic neutron scattering, is entirely IR and Raman inactive in vacuum. This is the mode responsible for the $282 \mathrm{~cm}^{-1}$ (vacuum), $318 \mathrm{~cm}^{-1}$ (cubic), 300-310 $\mathrm{cm}^{-1}$ (tetragonal), 370-372 $\mathrm{cm}^{-1}$ (orthorhombic) vibration. Progressive confinement of MA from vacuum to the orthorhombic phase blueshifts the energy of the vibration.
In the solid state, the degeneracies in the molecular modes are typically split by local environment effects, peaks are both redshifted and blueshifted, and the IR and Raman activity varies. As such, it is evident that analysis of the Raman and IR spectra in the experimentally easily accessible molecular frequency range can enable statements to be made about the local structure and configuration of the hybrid perovskite. Our data is collected for a particular representation of the cubic and tetragonal phase; in reality, the location of the MA in these phases will be disordered. As such, a detailed comparison of theory to experiment will require sampling the thermodynamic ensemble of structures. 


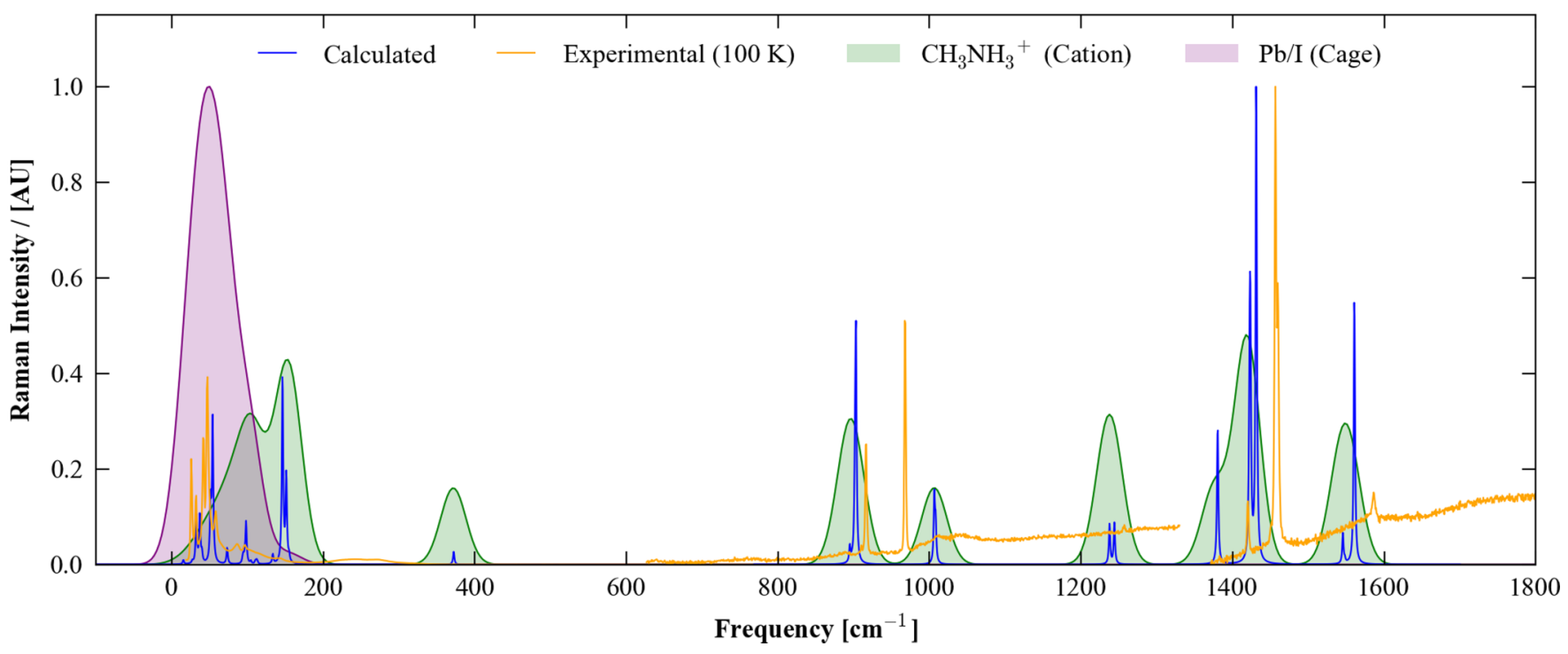

FIG. 3. (Color online) Comparison of the calculated and measured (100 K) Raman spectrum for the orthorhombic perovskite phase of $\mathrm{CH}_{3} \mathrm{NH}_{3} \mathrm{PbI}_{3}$ in the range 0-1800 $\mathrm{cm}^{-1}$, which includes phonon modes centered on the inorganic cage (lowest frequency), organic cage (highest frequency), and coupled modes (intermediate frequency). The simulated spectrum was broadened by convolution of a $2 \mathrm{~cm}^{-1}$ Lorentzian, while the underlying phonon density of states is shown for comparison with a broadening of $32 \mathrm{~cm}^{-1}$.

Here, Raman spectra for the three phases is reported across the full frequency range. The spectrum up to $450 \mathrm{~cm}^{-1}$ was reported in Ref. [46]. Reliable measurements are a challenge due to chemical instability of the material. $\mathrm{MAPbI}_{3}$ is strongly affected by environmental conditions, such as the presence of ambient moisture $[27,48]$. Isolated in vacuum, the material can still decompose and bleach due to heating, including by that imposed by the (typically high) Raman laser fluence [45]. Such degradation leads to the formation of $\mathrm{PbI}_{2}$, which overlaps in Raman spectra with $\mathrm{MAPbI}_{3}$, and so easily leads to misinterpretation.

The Raman spectra of a high-quality single crystal of $\mathrm{MAPbI}_{3}$ is shown in Fig. 2, and compared in detail with the calculations in Fig. 3. Across the full spectral range, the agreement between the predicted and measured spectra is good, with the response across bands (i)-(iii) well reproduced. On closer inspection of Fig. 3, there are noticeable shifts in peak positions, which can be attributed to three potential sources of error: (a) the harmonic approximation (anharmonic renormalization may be large), (b) the limits of the exchange-correlation treatment (nonlocal interactions may be important), and (c) the assumption of a fully ordered structure (local inhomogeneity may be prevalent). There is also a notable case of an absence of measured Raman activity around $150 \mathrm{~cm}^{-1}$, which we can tentatively attribute to a lifetime broadening effect. The same level of theory applied to the lead-based semiconductors $\mathrm{PbS}$ and $\mathrm{PbTe}$ results in quantitative agreement with measured phonon frequencies and dispersion [49], which highlights the complex nature of $\mathrm{CH}_{3} \mathrm{NH}_{3} \mathrm{PbI}_{3}$.

We have also included in Fig. 2 a room-temperature IR spectrum reported by Glaser et al. [35], which again shows excellent agreement across the spectrum. A number of very weak absorption peaks below $3000 \mathrm{~cm}^{-1}$ are evident, which could be related to molecular disorder and/or partial decomposition. The temperature-resolved (between 140 and 299 K) IR spectra for tetragonal and orthorhombic phases have been previously reported by Yamamuro [50]. We reproduce the position and the intensity of the peak observed at $900 \mathrm{~cm}^{-1}$, reliably assigning it to the (also Raman-active) $\mathrm{C}-\mathrm{N}$ bond stretch. High-quality IR measurements, in particular looking at the very low-energy transitions, would provide considerable information on the nature of the domains and local structure in a $\mathrm{MAPbI}_{3}$ film.

\section{Anharmonic effects}

The lattice dynamic simulations discussed above were performed within the harmonic approximation. All eigenmodes at the center of the Brillouin zone were real (positive frequencies) for each phase, i.e., the structures are locally stable.

The phonon dispersion across the first Brillouin zone is shown for the cubic perovskite structure in Fig. 4. Here imaginary (negative frequency or "soft") modes are found at the zone boundaries. Such instabilities are a common feature of the perovskite structure and represent antiferroelectric distortions linked to to rotations and tiling of the octahedra in neighboring unit cells [2]. The soft modes are centered around the $R$ and $M$ points, which correspond to the $\langle 111\rangle$ and $\langle 110\rangle$ directions in the cubic lattice. This behavior is similar to the inorganic perovskite $\mathrm{CsPCl}_{3}$, where neutron scattering was used to probe condensation of these modes, which leads to successive transitions from the cubic to tetragonal to orthorhombic phases [51]. The effect of these modes in $\mathrm{MAPbI}_{3}$, and the associated high levels of anharmonicity at room temperature, can be observed directly in molecular dynamics simulations, where temporal rotations of the $\mathrm{CH}_{3} \mathrm{NH}_{3}{ }^{+}$ions and distortions of the $\mathrm{PbI}_{6}$ octahedra have been found in several studies [22,28,52]. 

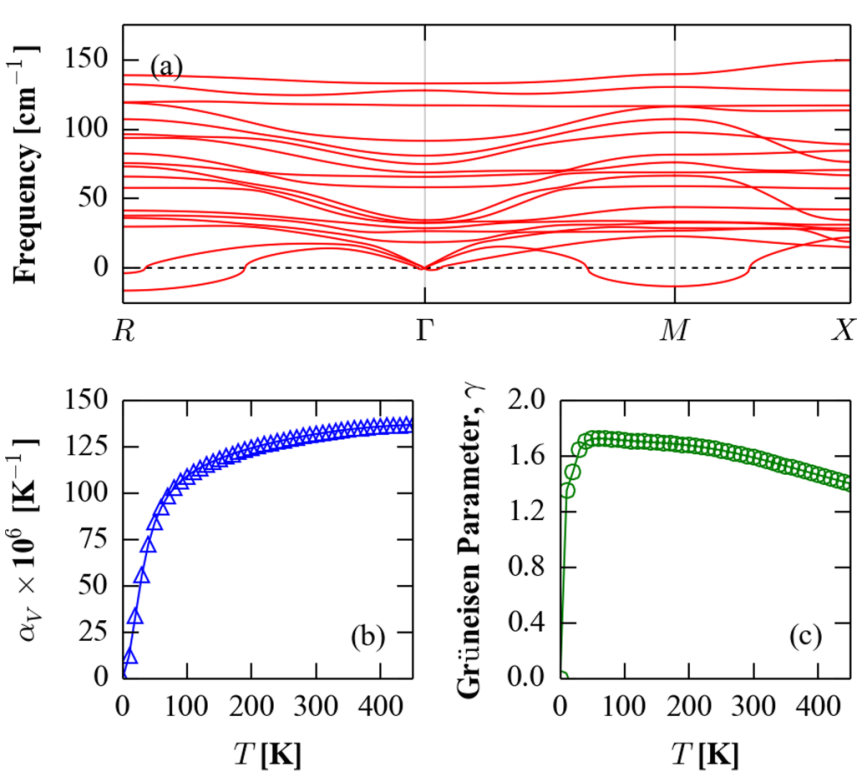

FIG. 4. (Color online) Vibrational properties of the cubic phase of $\mathrm{MAPbI}_{3}$ : (a) Phonon dispersion of the low-frequency (inorganic cage) modes within the harmonic approximation. Negative frequency (imaginary or "soft") modes are found at the Brillouinzone boundary points $M\left[\frac{2 \pi}{a}\left(\frac{1}{2}, \frac{1}{2}, 0\right)\right]$ and $R\left[\frac{2 \pi}{a}\left(\frac{1}{2}, \frac{1}{2}, \frac{1}{2}\right)\right]$. (b) Volumetric thermal expansion within the quasiharmonic approximation. (c) Average Grüneisen parameter within the quasiharmonic approximation.

An approach to include the effects of temperature (thermal expansion) and first-order anharmonicity in lattice dynamic calculations is the quasiharmonic approximation (QHA) [53,54]. The computational cost is one order of magnitude higher than the harmonic approximation and thus was considered for the cubic phase only. The volumetric thermal-expansion coefficient extracted from the PND data at $300 \mathrm{~K}$ is $1.32 \times 10^{-4} / \mathrm{K}$, which compares very well to the value of $1.25 \times 10^{-4} / \mathrm{K}$ computed within the QHA. The predicted thermal expansion for $\mathrm{MAPbI}_{3}$ is similar to inorganic semiconductors (e.g., for PbTe, the value is $0.7 \times$ $10^{-4} / \mathrm{K}$ at $\left.300 \mathrm{~K}[40]\right)$ and positive over the full temperature range.

The temperature dependence of the phonon modes can be described by the Grüneisen parameter, which has an average of around 1.6 (see Fig. 4), slightly below the value of 1.7 found in $\mathrm{PbI}_{2}$ [55]. The imaginary modes at $R$ and $M$ remain at all temperatures, consistent with the cubic lattice being a dynamic average of a locally distorted structure; the same phenomenon is observed in $\mathrm{CsSnI}_{3}$ [56]. The high level of anharmonicity associated with the soft tilting modes is consistent with the "ultralow" $\left(<1 \mathrm{Wm}^{-1} \mathrm{~K}^{-1}\right.$ at $\left.300 \mathrm{~K}\right)$ lattice thermal conductivity reported for single crystals and polycrystalline $\mathrm{MAPbI}_{3}$ [57]. Hybrid halide perovskites are thus also promising for application in thermoelectric devices if thermal-stability issues can be overcome [58].

\section{CONCLUSIONS}

The vibrational frequencies of three crystallographic phases of the hybrid perovskite $\mathrm{CH}_{3} \mathrm{NH}_{3} \mathrm{PbI}_{3}$ have been investigated. We identified three main phonon branches present in the three phases. Two high-frequency branches are associated with the vibration and bond stretching of the molecular cation with frequencies in the range 300 to $3300 \mathrm{~cm}^{-1}$. The lowest-energy branch, below $150 \mathrm{~cm}^{-1}$, arises predominately from the inorganic cage, but with half of the modes coupled to the motion of the molecule. The simulated Raman spectrum of the orthorhombic phase is in good agreement with measurements on a single crystal of $\mathrm{MAPbI}_{3}$. Dynamic instabilities occur at the zone boundaries of the cubic phase, which requires methods beyond the harmonic approximation, such as selfconsistent phonon theory, for an accurate treatment. These results suggest that the room-temperature structure of $\mathrm{MAPbI}_{3}$ is fluctional, owing to the persistent tilting and distortion of the octahedral networks and rotations of the molecular cations.

These factors may be important for developing a quantitative understanding and model of how hybrid perovskite solar cells operate. Upon excitation, the relative stability of free carriers and excitons depends intimately on the dielectric screening of the material, which includes vibrational and rotational components. The transport and recombination of photogenerated charge carriers will also be influenced by electron-phonon coupling, which can significantly reduce the effective size and distribution of electrons and holes within the perovskite layer.

Note added in proof. A neutron scattering investigation of $\mathrm{MAPbBr}_{3}$ has very recently been reported [59], which identifies strong coupling between the vibrations of the molecule and the inorganic cage, analogous to the overlap identified in our study of the iodide material. A soft mode associated with octahedral titling is also measured that is in support of our observations.

\section{ACKNOWLEDGMENTS}

The authors are grateful for helpful discussions with Mariano Campoy-Quiles. The research at the University of Bath has been supported by the EPSRC (Grants No. EP/K016288/1, No. EP/K004956/1, and No. EP/M009580/1), ERC (Grant No. 277757), EU-FP7 (Grant No. 316494), and the Royal Society. A.J.J. and O.J.W. were funded through the CDT in Sustainable Chemical Technologies (EPSRC Grant No. EP/ G03768X/1). P.R.F.B. and A.M.A.L. are grateful to the EPSRC (Grants No. EP/J002305/1, No. EP/M014797/1, and No. EP/M023532/1). A.R.G. acknowledges the Spanish Ministerio de Economa y Competitividad (MINECO) through Projects No. MAT201237776 and No. CSD2010-00044 (Consolider NANOTHERM). This work benefited from access to both the University of Bath's High Performance Computing Facility and ARCHER, the United Kingdom's national high-performance computing service, which is funded by the Office of Science and Technology through EPSRC's High End Computing Programme (Grant No. EP/L000202). 
[1] A. M. Glazer, Acta Crystallogr. Sect. B 28, 3384 (1972).

[2] N. A. Benedek and C. J. Fennie, J. Phys. Chem. C 117, 13339 (2013).

[3] D. B. Mitzi, J. Chem. Soc. Dalt. Trans. 2001, 1 (2001).

[4] J. M. Frost, K. T. Butler, F. Brivio, C. H. Hendon, M. Van Schilfgaarde, and A. Walsh, Nano Lett. 14, 2584 (2014).

[5] A. B. Cairns and A. L. Goodwin, Chem. Soc. Rev. 42, 4881 (2013).

[6] W. Li, Z. Zhang, E. G. Bithell, A. S. Batsanov, P. T. Barton, P. J. Saines, P. Jain, C. J. Howard, M. A. Carpenter, and A. K. Cheetham, Acta Mater. 61, 4928 (2013).

[7] G. Kieslich, S. Kumagai, K. T. Butler, T. Okamura, C. H. Hendon, S. Sun, M. Yamashita, A. Walsh, and A. K. Cheetham, Chem. Commun. 51, 15538 (2015).

[8] A. Kojima, K. Teshima, Y. Shirai, and T. Miyasaka, J. Am. Chem. Soc. 131, 6050 (2009).

[9] J.-H. Im, C.-R. Lee, J.-W. Lee, S.-W. Park, and N.-G. Park, Nanoscale 3, 4088 (2011).

[10] M. M. Lee, J. Teuscher, T. Miyasaka, T. N. Murakami, and H. J. Snaith, Science 338, 643 (2012).

[11] M. Liu, M. B. Johnston, and H. J. Snaith, Nature (London) 501, 395 (2013).

[12] F. De Angelis, Acc. Chem. Res. 47, 3349 (2014).

[13] J. Even, L. Pedesseau, C. Katan, M. Kepenekian, J.-S. Lauret, D. Sapori, and E. Deleporte, J. Phys. Chem. C 119, 10161 (2015).

[14] A. Walsh, D. O. Scanlon, S. Chen, X. G. Gong, and S.-H. Wei, Angew. Chemie Int. Ed. 54, 1791 (2015).

[15] A. Walsh, J. Phys. Chem. C 119, 5755 (2015).

[16] N. J. Jeon, J. H. Noh, W. S. Yang, Y. C. Kim, S. Ryu, J. Seo, and S. I. Seok, Nature (London) 517, 476 (2015).

[17] D. Weber, Z. Naturforsch. B 33b, 1443 (1978).

[18] N. Onoda-Yamamuro, T. Matsuo, and H. Suga, J. Phys. Chem. Solids 51, 1383 (1990).

[19] Y. Kawamura, H. Mashiyama, and K. Hasebe, J. Phys. Soc. Japan 71, 1694 (2002).

[20] N. Onoda-Yamamuro, T. Matsuo, and H. Suga, J. Phys. Chem. Solids 53, 935 (1992).

[21] M. T. Weller, O. J. Weber, P. F. Henry, A. M. Di Pumpo, and T. C. Hansen, Chem. Commun. 51, 4180 (2015).

[22] A. M. A. Leguy, J. M. Frost, A. P. McMahon, V. G. Sakai, W. Kochelmann, C. Law, X. Li, F. Foglia, A. Walsh, B. C. O’Regan, J. Nelson, J. T. Cabral, and P. R. F. Barnes, Nat. Commun. 6, 7124 (2015)

[23] A. A. Bakulin, O. Selig, H. J. Bakker, Y. L. A. Rezus, C. Müller, T. Glaser, R. Lovrincic, Z. Sun, Z. Chen, A. Walsh, J. M. Frost, and T. L. C. Jansen, J. Phys. Chem. Lett. 6, 3663 (2015).

[24] J. P. Perdew, A. Ruzsinszky, G. I. Csonka, O. A. Vydrov, G. E. Scuseria, L. A. Constantin, X. Zhou, and K. Burke, Phys. Rev. Lett. 100, 136406 (2008).

[25] See Supplemental Material at http://link.aps.org/supplemental/ 10.1103/PhysRevB.92.144308 for an animation of the zonecenter vibrational modes of the cubic perovskite phase and a comparison of the simulated and measured diffraction patterns.

[26] A. Poglitsch and D. Weber, J. Chem. Phys. 87, 6373 (1987).

[27] T. Baikie, Y. Fang, J. M. Kadro, M. Schreyer, F. Wei, S. G. Mhaisalkar, M. Gratzel, and T. J. White, J. Mater. Chem. A 1, 5628 (2013).

[28] J. M. Frost, K. T. Butler, and A. Walsh, APL Mater. 2, 081506 (2014).
[29] https://github.com/WMD-Bath/Hybrid-perovskites.

[30] R. Wasylishen, O. Knop, and J. Macdonald, Solid State Commun. 56, 581 (1985).

[31] Z. Fan, J. Xiao, K. Sun, L. Chen, Y. Hu, J. Ouyang, K. P. Ong, K. Zeng, and J. Wang, J. Phys. Chem. Lett. 6, 1155 (2015).

[32] C. Quarti, E. Mosconi, and F. De Angelis, Chem. Mater. 26, 6557 (2014).

[33] R. J. Worhatch, H. J. Kim, I. P. Swainson, a. L. Yonkeu, and S. J. L. Billinge, Chem. Mater. 20, 1272 (2008).

[34] F. Brivio, A. B. Walker, and A. Walsh, APL Mater. 1, 042111 (2013).

[35] T. Glaser, C. Müller, M. Sendner, C. Krekeler, O. E. Semonin, T. D. Hull, O. Yaffe, J. S. Owen, W. Kowalsky, A. Pucci, and R. Lovrinčić, J. Phys. Chem. Lett. 6, 2913 (2015).

[36] G. Kresse and J. Furthmüller, Comput. Mater. Sci. 6, 15 (1996).

[37] G. Kresse and D. Joubert, Phys. Rev. B 59, 1758 (1999).

[38] P. E. Blöchl, Phys. Rev. B 50, 17953 (1994).

[39] A. Togo, L. Chaput, I. Tanaka, and G. Hug, Phys. Rev. B 81, 174301 (2010).

[40] J. M. Skelton, S. C. Parker, A. Togo, I. Tanaka, and A. Walsh, Phys. Rev. B 89, 205203 (2014).

[41] A. Togo, L. Chaput, and I. Tanaka, Phys. Rev. B 91, 094306 (2015).

[42] R. P. Stoffel, C. Wessel, M.-W. Lumey, and R. Dronskowski, Angew. Chem. Int. Ed. England 49, 5242 (2010).

[43] S. Baroni and S. D. Gironcoli, Rev. Mod. Phys. 73, 515 (2001).

[44] J. M. Skelton, E. L. da Silva, R. Crespo-Otero, L. E. Hatcher, P. R. Raithby, S. C. Parker, and A. Walsh, Faraday Discuss. 177, 181 (2015).

[45] M. Ledinský, P. Löper, B. Niesen, J. Holovský, S.-J. Moon, J.-H. Yum, S. De Wolf, A. Fejfar, and C. Ballif, J. Phys. Chem. Lett. 6, 401 (2015).

[46] C. Quarti, G. Grancini, E. Mosconi, P. Bruno, J. M. Ball, M. M. Lee, H. J. Snaith, A. Petrozza, and F. D. Angelis, J. Phys. Chem. Lett. 5, 279 (2014).

[47] T. Ahmed, C. La-o vorakiat, T. Salim, Y. M. Lam, E. E. M. Chia, and J.-X. Zhu, Europhys. Lett. 108, 67015 (2014).

[48] J. H. Noh, S. H. Im, J. H. Heo, T. N. Mandal, and S. I. Seok, Nano Lett. 13, 1764 (2013).

[49] J. M. Skelton, D. Tiana, S. C. Parker, A. Togo, I. Tanaka, and A. Walsh, J. Chem. Phys. 143, 064710 (2015).

[50] N. Onoda-yamamuro, Ph.D. thesis, Osaka University, 1992.

[51] Y. Fujii, S. Hoshino, Y. Yamada, and G. Shirane, Phys. Rev. B 9, 4549 (1974).

[52] C. Quarti, E. Mosconi, and F. De Angelis, Phys. Chem. Chem. Phys. 17, 9394 (2015).

[53] M. T. Dove, Am. Mineral. 82, 213 (1997).

[54] J. Buckeridge, D. O. Scanlon, A. Walsh, C. R. A. Catlow, and A. A. Sokol, Phys. Rev. B 87, 214304 (2013).

[55] W. M. Sears, M. L. Klein, and J. A. Morrison, Phys. Rev. B 19, 2305 (1979).

[56] E. L. da Silva, J. M. Skelton, S. C. Parker, and A. Walsh, Phys. Rev. B 91, 144107 (2015).

[57] A. Pisoni, J. Jaćimović, O. S. Barišić, M. Spina, R. Gaál, L. Forró, and E. Horváth, J. Phys. Chem. Lett. 5, 2488 (2014).

[58] Y. He and G. Galli, Chem. Mater. 26, 5394 (2014).

[59] I. P. Swainson, C. Stock, S. F. Parker, L. Van Eijck, M. Russina, and J. W. Taylor, Phys. Rev. B 92, 100303(R) (2015). 